Mini Review

\title{
Phospholipid transport and remodeling in health and disease
}

\author{
Katarína Poloncová and Peter Griač \\ Institute of Animal Biochemistry and Genetics, Slovak Academy of Sciences, Moyzesova 61, 90028 Ivanka pri Dunaji, \\ Slovak Republic
}

\begin{abstract}
Lipids not only form the backbone of biological membranes, but also serve as the source of numerous regulatory and signaling molecules. Understanding the role of lipids in the physiology of eukaryotic cell will help to identify mechanisms behind lipid-related human diseases. This minireview concentrates on two examples of human diseases associated with phospholipid remodeling and transport. The first is Barth syndrome, a severe rare genetic disorder. Barth syndrome is the first recognized human disease in which the primary causative factor is a defective remodeling of the signature mitochondrial phospholipid cardiolipin. The other example involves defects associated with lipid transfer proteins (LTPs). LTPs regulate diverse lipid-mediated cellular processes important for maintaining the specific composition of different cellular organelles. In vitro LTPs facilitate lipid transport between membranes through an aqueous environment. This article is not intended to be a comprehensive review of lipid-related human diseases; its aim is rather to stress the importance of basic lipid research in our advancement in the diagnosis and treatment of diseases.
\end{abstract}

Key words: Phospholipid - Cardiolipin — Signaling - Phosphatidylinositol transfer protein - Barth syndrome

\begin{abstract}
Abbreviations: AAC, ATP/ADP carrier; CDP-choline, cytidine diphosphate choline; CL, cardiolipin; CoA, coenzyme A; CRAL_TRIO domain, cellular retinaldehyde-binding/triple function domain; GAPs, GTPase activating proteins; GEFs, guanidine nucleotide exchange factors; GTP, guanidine triphosphate; LTPs, lipid transfer proteins; PC, phosphatidylcholine; PE, phosphatidylethanolamine; PI, phosphatidylinositol; PI-4-P, phosphatidylinositol-4-phosphate; PITP, phosphatidylinositol transfer protein; $\mathrm{PiC}$, phosphate carrier; ROS, reactive oxygen species.
\end{abstract}

\section{Introduction}

Biological membranes form a protective barrier around cells and their organelles. In addition, the biological membrane is the place where many important cellular processes take place. The proper functioning of this extremely thin layer of lipids, proteins and carbohydrates is often a matter of life or death for the cell and the whole organism. Eukaryotic cells contain a large spectrum of lipid molecules (typically more than 1000) belonging to one of three categories: glycerophospholipids, sphingolipids, and sterols. This abundance of diverse lipid

Correspondence to: Peter Griač, Institute of Animal Biochemistry and Genetics, Slovak Academy of Sciences, Moyzesova 61, 90028 Ivanka pri Dunaji, Slovak Republic

E-mail: Peter.Griac@savba.sk molecules suggests that lipids are more than just the building blocks of membrane bilayers or energy stores for an organism. Lipids serve also as precursors of steroid hormones, signaling molecules, and second messengers. They are involved in intermembrane transport and cell cycle regulation. Indeed, we are only starting to realize the nature of membrane lipid complexity. Further progress in the field of membrane lipidology will not only help to quench our curiosity in understanding the basic principles of how cells and organisms operate, but may also provide insights into membrane-related human diseases. This minireview describes examples of human membrane lipid-related diseases associated with phospholipid remodeling and transport. Lipid remodeling and intracellular transport are two important processes contributing to specific membrane lipid composition of eukaryotic cells which have not been fully explored and only recently are subjects of intensive study. 


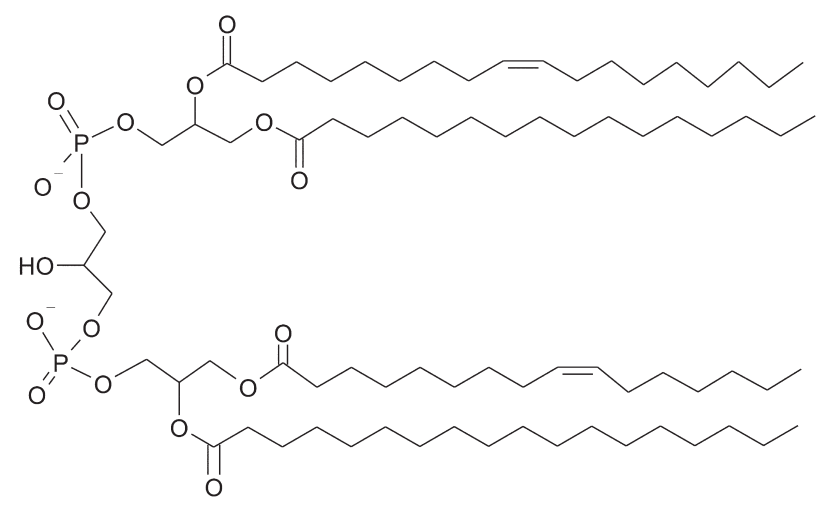

Figure 1. Scheme of cardiolipin.

\section{Barth syndrome - defective cardiolipin remodeling is the culprit}

Cardiolipin (CL), also called diphosphatidylglycerol, is a unique dimeric phospholipid that carries four acyl groups and two negative charges (Fig. 1). CL, due to its ability to form non-bilayer structures, is important in membrane domains of high curvature such as mitochondrial cristae (Chernomordik and Kozlov 2005; Chernomordik et al. 2006). It is almost exclusively associated with membranes generating an electrochemical gradient used to produce ATP in prokaryotes and eukaryotes alike (Koshkin and Greenberg 2000). Such membranes include the bacterial plasma membrane and the inner membrane of mitochondria. CL acts as a "flexible linker" that fills in cavities at protein interfaces and stabilizes individual subunits of oligomeric proteins and protein supercomplexes (Zhang et al. 2002; Pfeiffer et al. 2003). Respiratory complexes I, III, IV and V (the key components of the mitochondrial oxidative phosphorylation chain), two inner mitochondrial membrane carriers, ATP/ADP carrier (AAC) and phosphate carrier $(\mathrm{PiC})$, as well as two peripheral proteins, cytochrome $c$ and creatine kinase, all bind CL with high efficiency (Schlame et al. 2000). Although CL is not strictly required for the function of any of the respiratory chain supercomplexes, it increases their efficiency significantly (Jiang et al. 2000; Koshkin and Greenberg 2000; Claypool et al. 2008). At least three enzymatic reactions are involved in the production of mature CL: i) de novo synthesis catalyzed by cardiolipin synthase, ii) deacylation of CL to monolysoCL catalysed by mitochondrial phospholipase A, and iii) reacylation of monolyso-CL by a specific transacylase called tafazzin (Fig. 2). Deacylation of premature cardiolipin and subsequent reacylation are important catalytical steps leading to fully functional CL. Both, the amount of CL and the presence of specific CL species are critical for optimal functioning of mitochondria. In mammals, newly synthesized CL is rapidly remodeled to generate CL species enriched in linoleic acid, an unsaturated $18: 2$ fatty acid (Hauff and Hatch 2006). In yeast mono-unsaturated acyl chains 16-18 carbons long are the most prevalent ones (Chen et al. 2008). Changes in CL homeostasis have been described in various pathologies including ischemia and reperfusion, aging, heart failure, thyroid function, Parkinson's disease, diabetes and chronic ethanol consumption (Chicco and Sparagna 2007). Loss of CL and its peroxidation lead to the detachment of cytochrome $c$ from the mitochondrial membrane and its transfer into the cytosol. This change of cytochrome $c$ localization triggers the early stages of apoptosis (Wang 2001; Macchioni et al. 2010).

Recently, it was shown that aberrant CL metabolism, particularly its remodeling, is associated with a severe genetic disorder, Barth syndrome (Vreken et al. 2000; Schlame et al. 2002). Barth syndrome is a rare X-linked recessive cardioskeletal myopathy and neutropenia with typical symptoms of heart failure, myopathy, neuropathy and growth defects that was first described by Peter Barth and his colleagues. The clue to the underlying mechanism of the disease was abnormal mitochondria present in patients' tissues (Barth et al. 1983). Biochemically, patients have elevated urinary levels of 3-methylglutaconate, 3methylglutarate, and 2-ethylhydracrylate and suffer from hypocholesterolemy (Kelley et al. 1991). Both in fibroblasts and muscle biopsy samples respiratory chain abnormalities were observed. The patients share distinct facial features (tall and broad forehead, round face with prominent chin and full cheeks, large ears, and deep-set eyes), which are most evident in infancy (Hastings et al. 2009). They have cognitive difficulties with regard to mathematics, visual spatial tasks, and short-term memory. Language ability is spared. The prevalence of Barth syndrome in the population is not precisely known but the disease is likely to be under diagnosed. This could be due to poor diagnostics and large variability of the disease phenotype (pathology as well as biochemistry) (Spencer et al. 2005; Steward et al. 2010). In some patients almost normal urinary levels of 3-methylglutaconate were observed. Little correlation between severity of the disease and 3-methylglutaconate excretion is observed (Schmidt et al. 2004), and a high variability in 3-methylglutaconate excretion, even within 24 hours, contribute to the problems with Barth syndrome diagnosis (Christodoulou et al. 1994; Cantlay et al. 1999). In addition, neutropenia observed in patients with Barth syndrome may be intermittent (Barth et al. 1999), or many of the symptoms may be ascribed to acute viral insult. While some authors report that the disorder occurs between 1 to 49 years and its incidence is highest in early puberty (Barth et al. 2004), others claim that Barth syndrome may result in miscarriages, stillbirths and early neonatal death in male fetuses (Steward et al. 2010). The carrier mothers 


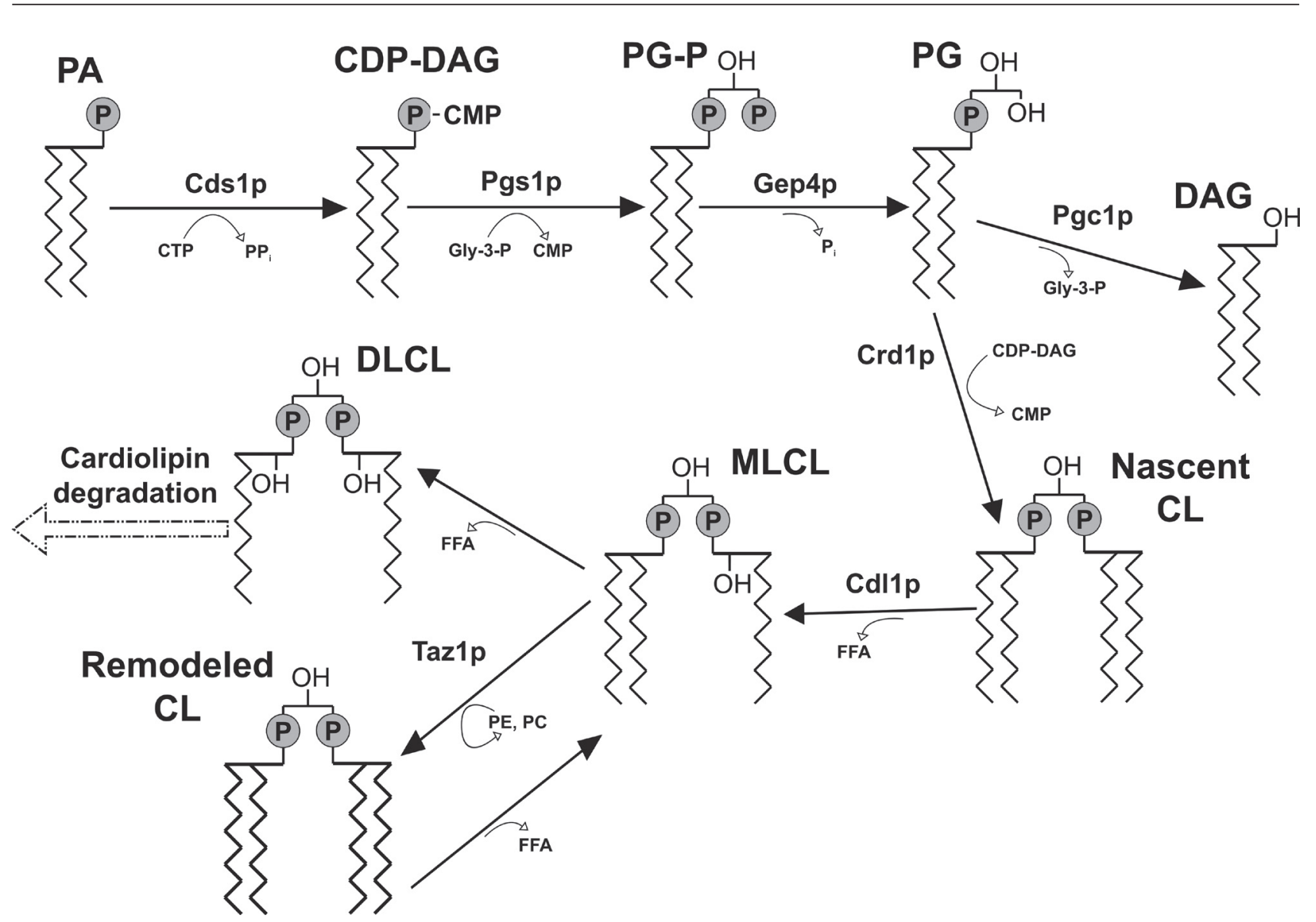

Figure 2. Metabolic pathways for cardiolipin biosynthesis and degradation. PA, phosphatidic acid; CDP-DAG, cytidine diphosphate diacylglycerol; PG-P, phosphatidylglycerol phosphate; PG, phosphatidylglycerol; DAG, diacylglycerol; nascent CL, cardiolipin before remodeling; MLCL, monolysocardiolipin; remodeled CL, cardiolipin with modified acyl chains - mostly unsaturated; DLCL, dilysocardiolipin; CTP, cytidine triphosphate; CMP, cytidine monophosphate; Gly-3-P, glycerol-3-phosphate; $\mathrm{PP}_{\mathrm{i}}$, diphosphate; $\mathrm{P}_{\mathrm{i}}$, phosphate; FFA, free fatty acids; PC, phosphatidylcholine; PE, phosphatidylethanolamine; Cds1p, CDP-diacylglycerol synthase; Pgs1p, phosphatidylglycerolphosphate synthase; Gep4p, mitochondrial phosphatidylglycerophosphatase; Pgclp, phosphatidylglycerol phospholipase C; Crd1p, cardiolipin synthase; Cdl1p, cardiolipin specific deacylase with phospholipase A2 activity; Tazlp, CoA independent lysophosphatidylcholine acyltransferase/tafazzin.

remain asymptomatic (Steward et al. 2010) which further complicates effective diagnosis.

Genetic linkage studies demonstrated that the gene TAZ (G4.5) was responsible for Barth syndrome when mutated (Bione et al. 1996). This gene and its various homologues in eukaryotes form a large superfamily of putative phospholipid acyltransferases. TAZ encodes a mitochondrial CoA-independent phospholipid-lysophospholipid transacylase required for CL homeostasis. It has no phospholipase activity (Xu et al. 2006b). Its deficiency drastically alters CL composition in humans (Vreken et al. 2000; Schlame et al. 2002), flies (Xu et al. 2006b), and yeast (Gu et al. 2004). There is considerable variation in type and localization of mutations leading to the manifestation of Barth syndrome (Spencer et al. 2005). This large variability has no obvious correlation with the disease phenotype and its severity. Even patients from the same families carrying the same mutations display variable symptoms and severity of the disease (Johnston et al. 1997). In humans, tafazzin deficiency leads to diversification of the CL species, accumulation of monolysoCL at the expense of the dominant species of tetra-linoleoyl CL, and decline of the total CL content. This specific form of CL deficiency was found to be associated with abnormalities of the mitochondrial inner membrane cristae (Vreken et al. 2000; Vaz et al. 2003; Gu et al. 2004; Ma et al. 2004; Xu et al. 2006a). Due to the similar effects of tafazzin deficiency, Drosophila and yeast were found to be useful models in determining the role of tafazzin in CL metabolism and its connection to Barth syndrome in humans. Studies in Drosophila helped not only to uncover the 
function of taffazin as a CoA-independent transacylase (Xu et al. 2006b), but also showed that changes in CL content and composition in fly Barth syndrome model were associated with the presence of abnormal mitochondria, especially in tissues where mitochondria with highly organized and densely packed cristae are present. Associated phenotypes are most pronounced under stress conditions and under conditions when active mitochondrial biogenesis and high energy production are required. This may explain why under normal conditions some Barth syndrome patients may remain largely asymptomatic with their symptoms often deteriorating during infections, growth spurt and other episodes of physiological strain (Acehan et al. 2009). Yeast taz1 mutants have altered CL acyl composition and multiple mitochondrial abnormalities when compared to the wild type strain. A typical feature of these mutants is also accumulation of monolyso-CL ( $\mathrm{Li}$ et al. 2007). The above mentioned observations are consistent with the role of Tazlp as a transacylase operational in a two-step process in which newly synthesized CL is deacylated to form monolyso-CL, which is then reacylated to mature CL. Deacylation of premature CL and formation of monolysoCL in yeast is catalysed by mitochondrial phospholipase Cld1p (Beranek et al. 2009). Importantly, the human TAZ gene was able to complement taz1 $\Delta$ associated defects in yeast, confirming that yeast taz $1 \Delta$ mutant is an appropriate Barth syndrome model. Later, it was described that Tazlp associates with the ATP synthase complex and also with the inner mitochondrial membrane transporter Aac2p, whose function depends on CL (Claypool et al. 2008). CL, because of its high proportion of unsaturated fatty acids, is highly susceptible to peroxidation (Imai et al. 2003). In taz1 $\Delta$ and $c r d 1 \triangle$ (CRD1 gene encodes for CL synthase) mutants with decreased levels of CL, toxicity of ethanol at higher temperatures and increased protein carbonylation in the presence of ethanol was observed (Chen et al. 2008). This observation correlates with reactive oxygen species (ROS) production in mitochondria and the resulting oxidative stress (Costa et al. 1997; Du and Takagi 2007). Excess ROS is associated with a variety of diseases and aging (Costa and Moradas-Ferreira 2001). It is likely that increased oxidative stress in yeast CL-deficient mutants in the presence of ethanol is caused by destabilization of protein complexes of the mitochondrial respiratory chain. For example, destabilized complex III, which is the major source of superoxide in mitochondria (Chance et al. 1979; Chen et al. 2003), may leak electrons to oxygen, constituting the primary source of superoxide anion (Turrens 2003). Increased toxicity of ethanol in these mutants can be eliminated by adding oleic acid to the growth media (Chen et al. 2008). These data indicate that CL remodeling, catalyzed by Tazlp, may have an additional important role in mitochondrial physiology - to help replace peroxidated acyl groups of CL.
Accumulated evidence from clinical and experimental studies using mammalian cells as well as fly and yeast model organisms support CL remodeling as a major defect in Barth syndrome. The presence of multiple TAZ transcripts and the variability of Barth syndrome phenotypes raise the possibility that other cellular functions could be also affected. Thus, future detailed investigations are likely to provide important insights into mechanisms of dysfunction associated with Barth syndrome and hopefully will identify potential targets for treating this disease.

\section{Lipid transfer proteins - location, location, location}

Lipid biosynthesis is restricted to a few compartments of eukaryotic cells, mostly the endoplasmic reticulum, mitochondria, lipid droplets, and Golgi apparatus (Fig. 3) - and yet the majority of lipid species are found in all cellular membranes. This necessitates the existence of efficient mechanisms of intracellular lipid transport. Traditionally, four ways for the transfer of lipids between membranes have been considered: i) spontaneous diffusion through the aqueous environment, ii) protein-mediated transfer catalyzed by lipid transfer proteins, iii) vesicle flux delivering membrane material from donor to an acceptor compartment, iv) lipid transfer at membrane contact sites (Holthuis and Levine 2005) (Fig. 4). Spontaneous lipid desorption from the membrane and lipid diffusion through the aqueous environment in the monomeric form is extremely slow for most classes of lipids. As the rate of spontaneous lipid exchange correlates with lipid aqueous-phase solubility lyso-phospholipids (for example lyso-PC) (McLean and Phillips 1984) exchange more rapidly than phospholipids. In general, this process is too slow to account for observed substantial non-vesicular lipid transport of most lipids (reviewed in Lev 2010). Lipid transfer proteins (LTPs) can greatly facilitate lipid transport between membranes. LTPs were identified in in vitro biochemical assays in which transport of hydrophobic molecules between two membranes through the aqueous compartment was dependent on the presence of specific cytosolic proteins (Wirtz 1991). A typical feature of the LTP structure is the existence of a lipid-binding pocket lined with mostly hydrophobic residues that stabilize the internalized lipid. In this way the hydrophobic lipid molecule is shielded from the water environment. Examples of hydrophobic molecules that can be sequestered by LTPs include not only lipids but also other lipophilic molecules. Typical substrates of LTPs are: phosphatidylinositol (PI), phosphatidylcholine (PC), phosphatidylethanolamine $(\mathrm{PE})$, cholesterol, oxysterols, ceramides, retinol and $\alpha$ tocopherol. In addition to the hydrophobic substrates they transport, LTPs are grouped into families based on 


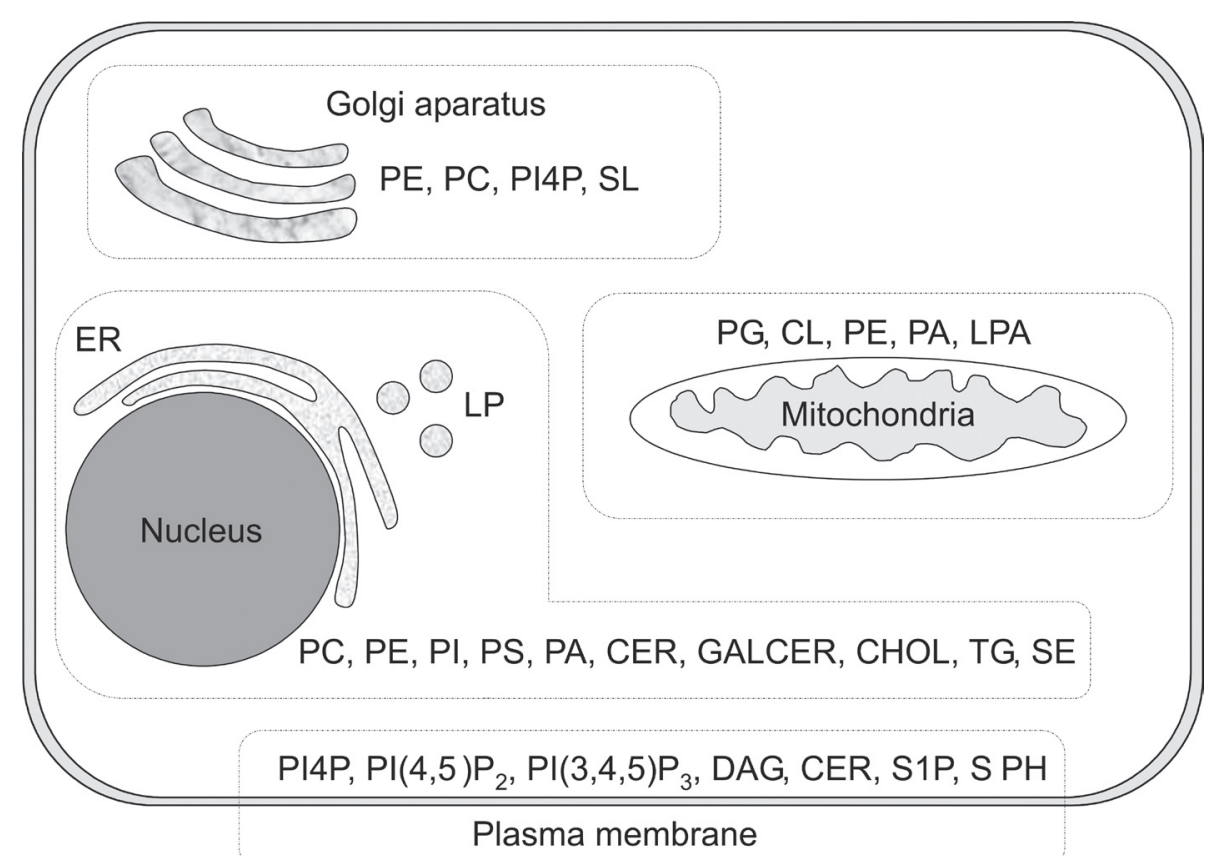

Figure 3. Compartmentalization of eukaryotic lipid biosynthesis. In the centre of mammalian lipid biosynthesis sits the endoplasmic reticulum (ER) from which lipids and their precursors are moved to lipid biosynthetic systems in other organelles. Note that in the plasma membrane no de novo lipid synthesis takes place. All the depicted lipid molecules generated in the plasma membrane are products of degradation and turnover of lipids synthesized elsewhere in the cell. PC, phosphatidylcholine; PE, phosphatidylethanolamine; PI, phosphatidylinositol; PS, phosphatidylserine; PA, phosphatidic acid; PG, phosphatidylglycerol; CL, cardiolipin; LPA, lysophosphatidic acid; DAG, diacylglycerol; PI4P, phosphatidylinositol-4-phosphate; $\mathrm{PI}(4,5) \mathrm{P}_{2}$, phosphatidylinositol-4,5-bisphosphate; $\mathrm{PI}(3,4,5) \mathrm{P}_{3}$, phosphatidylinositol-3,4,5-trisphosphate; CER, ceramide; GALCER, galactosylceramide; TAG, triacylglycerol; CHOL, cholesterol; SE, steryl esters; SL, sphingolipids; S1P, sphingosine-1-phosphate; SPH, sphingosine.

the three-dimensional structures of their lipid-binding domains (Cockcroft and Carvou 2007).

Lipid transport, and more specifically lipid transporters, are important for human health. Precise understanding on the molecular level of how lipid transfer proteins function is important for the understanding of the etiology of lipidtransfer related human diseases and for developing new therapies. It seems that of the many LTP families, the proteins possessing the SEC14 domain, also known as CRAL_TRIO (cellular retinaldehyde-binding/triple function) domain, are the most common lipid transporters mutated in disease. For example, mutations in the gene for retinaldehyde-binding protein causes retinitis pigmentosa, the human disease characterized by inherited blindness (Maw et al. 1997). Another typical human disease connected to the Sec14 group of LTPs is ataxia, a neurodegenerative disease caused by mutations in the gene encoding a-tocopherol transfer protein (Ouahchi et al. 1995). Recently, mutations in the protein caytaxin containing the Sec14 domain were found to be responsible for another neurodegenerative disease, Cayman ataxia (Bomar et al. 2003). A Sec14-like lipid-binding domain is also found in the protein neurofibromin. Mutations in the gene encoding human neurofibromin predispose the individual to the development of a variety of tumors affecting mostly the peripheral and central nervous systems (Cichowski and Jacks 2001). Altogether, protein databases contain over 1500 proteins with the Sec14 domain, including at least 29 encoded by the human genome (http://www.ncbi.nlm.nih. gov/). Interestingly, in mammals, the Sec14 domain is often incorporated into complex proteins possessing a diversity of functions in addition to stand-alone Sec14-domain proteins, more typical for simple eukaryotes. Many of these complex mammalian Sec14 domain-containing proteins are guanine nucleotide exchange factors (GEFs) and GTPase activating proteins (GAPs). This suggests that at least some members of the Sec14 protein family have a regulatory role connected to $\mathrm{G}$ protein activity.

The prototype domain (CRAL_TRIO in mammalian proteins) is the yeast Saccharomyces cerevisiae PI transfer protein Sec14p (Bankaitis et al. 1990). The crystal structure of detergent-bound Sec14 protein has been solved (Sha et al. 1998). In addition, crystal structures of several phospholipid-bound forms of yeast Sfh1p (YKL091c), the protein with the highest degree of similarity to Sec14p, are now available 
(Schaaf et al. 2008). Crystal structures of some mammalian proteins containing Sec14-like domains are also known. These include $\alpha$-tocopherol transfer protein (Meier et al. 2003; Min et al. 2003) and neurofibromin (D'Angelo et al. 2006; Welti et al. 2007). All Sec14-like proteins or domains crystallized to date contain a hydrophobic lipid-binding cavity gated by a mobile helical structure, important for molecular dynamics of substrate exchange reactions on the surfaces of biological membranes (Bankaitis et al. 2010). The yeast Sec14p contains only the Sec14 domain without additional known functional domains. It is required for transport of secretory proteins from the Golgi apparatus and is essential for cell viability (Bankaitis et al. 1989, 1990). The ability of Sec14p to facilitate the transfer of PI and PC in vitro between biological and artificial membranes suggested a role in the intracellular trafficking of phospholipids. Accumulated genetic evidence now indicates that Sec14p regulates phospholipid metabolic pathways that have an impact on signaling and membrane trafficking in a complex way. This conclusion came from the analysis of genetic suppressors of the otherwise essential Sec14p requirement for Golgi function and cell viability. Suppressor (or "bypass") mutations in this case are thought to restore physiological conditions that are consequences of Sec14p function in vivo and are changed when this protein is non-functional. There is a clear connection between the function of Sec14p and PC metabolism. First, inactivation of any of the three structural genes encoding enzymes of the CDP-choline (or Kennedy) pathway for PC biosynthesis "bypass" the requirement of Sec14p for secretion and viability (Cleves et al. 1991). Second, inactivation of the other pathway leading to PC biosynthesis, the PE pathway, together with the inhibition of choline uptake from the medium, also leads to the rescue of cells lacking functional Sec14p (Xie et al. 2001). Third, in the absence of functional Sec14p increased turnover of PC via phospholipase D was observed (Sreenivas et al. 1998) and intact phospholipase D1 (encoded by the SPO14 gene) must be present for all genetic supressors to bypass the Sec14p requirement (Xie et al. 1998). Fourth, in the absence of functional Sec14p decreased PC turnover via intracellular phospholipase B, Nte1p, producing free fatty acids and glycerophosphocholine was observed (Murray and McMaster 2005). In addition to the regulation of PC metabolism, regulation of phosphoinositide metabolism represents another important regulatory role of Sec14p. One of the other genetic bypass suppressors of Sec14p requirement was the deletion of yeast PI 4-phosphatase, encoded by the SAC1 gene (Cleves et al. 1989). Moreover, later experiments revealed that in the mutant with the temperature-sensitive sec14-1 allele, when shifted to a non permissive temperature of $37^{\circ} \mathrm{C}$, levels of PI-4-phosphate (PI-4-P) at the Golgi were significantly reduced (Hama et al. 1999). Also, inactivation of the KES1 gene, which encodes an oxysterol-binding protein family member, resulted in the

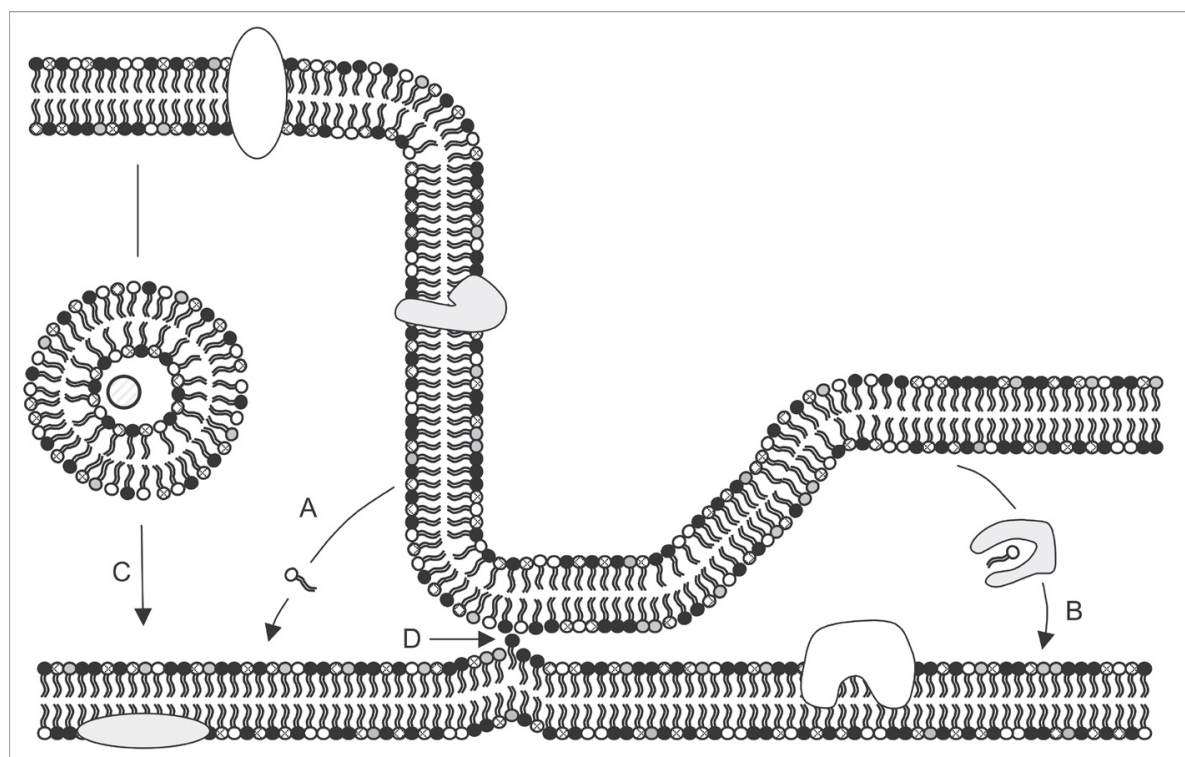

Figure 4. Schematic illustration of four major ways of intermembrane lipid transport. A. Spontaneous diffusion through the aqueous environment. B. Protein-mediated transfer catalyzed by lipid transfer proteins. C. Vesicular transport delivering membrane material from donor to an acceptor compartment. D. Lipid transfer at membrane contact sites. There are indications that some of these mechanisms operate simultaneously, e.g. lipid transfer proteins participating in lipid movement between membranes at their contact sites. 
ability of the yeast cell to survive in the absence of Sec14p (Fang et al. 1996). Recent high throughput genetics analysis revealed that Sec14p and Kes1 $\mathrm{p}$ are involved in coordinate regulation of the Golgi PI-4-P pool generated by Piklp, one of two essential phosphoinositide 4-kinases (LeBlanc and McMaster 2010).

In conlusion, the current view is that Sec14p coordinates lipid metabolism with membrane trafficking into and out of the trans-Golgi (Griac et al. 2006; Bankaitis et al. 2010; LeBlanc and McMaster 2010), providing the appropriate balance of lipids for this trafficking to occur. The molecular details of how Sec14p regulates membrane traffic is, however, unclear. One hypothesis presumes that Sec14-like proteins operate primarily as lipid sensors that instruct specific enzymes when and where to execute biochemical reactions (Bankaitis et al. 2010). This sensing information can be conveyed through spatially and temporary regulated changes in phosphoinositide metabolism dependent on Sec14p. To date, the mechanism of how Sec14-like proteins (at least some of them) can sense the membrane lipid composition is not clear and additional experiments are needed to test this hypothesis. The other possibility is that Sec14-like proteins operate at membrane contact sites where transfer of lipids can occur (Levine and Loewen 2006). An example of this mode of action by a Sec14-like protein is the yeast Sec14 protein homologue Pdr17p (also known as Sfh4p, or PstB2p). All available data strongly suggest that Pdr17p acts as a component of a multiprotein complex that effects transport of phosphatidylserine between endoplasmic reticulum and Golgi membranes at membrane contact sites (Wu et al. 2000; Wu and Voelker 2001). It is also possible that individual members of the Sec14-like superfamily of proteins differ in their mechanisms of function. Future advances in technology will hopefully enable us to follow individual lipid transfer proteins loaded with different cargo molecules as they go about their business in the cell and answer questions on how Sec14-like proteins participate in diverse biological processes linked to various aspects of cellular metabolism.

In higher eukaryotes, including mammals, PI transfer activity was identified in a group of PI transfer proteins (PITPs) that have no sequence or structural homology to the group of Sec14-like proteins. Thus, caution has to be exercised when comparing these two families of PI transfer proteins. Saying that, it is, however, also important to stress that yeast Sec14p and some mammalian PITPs functionally partially overlap. Yeast Sec14p is able to restore phospholipase $\mathrm{C}$ signaling in mammalian cells lacking PITPs (Cunningham et al. 1996) and vice versa: mammalian PITPs can rescue yeast cells with the temperature sensitive allele of the SEC14 gene (Tanaka and Hosaka 1994). The function of higher eukaryotes PITPs was studied in various model organisms, including mice, rats, flies, and zebrafish. In mice and Drosophila insufficiencies in PITPs lead to severe neurodegenerative defects (Hamilton et al. 1997; Milligan et al. 1997; Alb et al. 2003). In mammals, there are three soluble PITPs (PITP $\alpha$, PITP $\beta$ and $\operatorname{RdgB} \beta$ ), some of which are differentially spliced into variants with variations in the $\mathrm{C}$-terminal region, and two multidomain proteins (RdgBaI/Nir2 and RdgBaII/Nir3) containing the PITP domain (Cockcroft and Carvou 2007). All of these proteins are able to transfer PI between membranes through the aqueous phase in vitro (Cockcroft 1998). Two soluble mammalian PITPs, PITP $\alpha$ and PITP $\beta$, share sequence identity of $77 \%$ and a sequence similarity of $94 \%$. Despite this high sequence similarity it is now well established that these two isoforms serve different functions in the cell. First, they differ in subcellular location; PITP $\beta$ is present mostly at the Golgi compartment, and PITPa was found throughout the cytosol and in the nucleus (De Vries et al. 1996). Second, the phenotype of mutations in these PITPs is different. In mutant mice defective in PITPa function, called vibrator, progressive tremor and degeneration of neurons in brain and spinal cord were observed (Hamilton et al. 1997), which lead to juvenile death of these individuals. A more severe phenotype was observed in PITPa knockout mice. PITPa ${ }^{-/-}$mice died a few days after birth from complicated organ failure including spinocerebellar disease, hypoglycemia and intestinal and hepatic steatosis (Alb et al. 2003). In contrast, ablation of the gene for PITP $\beta$ leads to early embryonic lethality (Alb et al. 2002). Another neuronal phenotype, retinal neurodegeneration was first observed in fruit fly Drosophila following disruption of RdgBa (Milligan et al. 1997).

The three-dimensional structure of PITP bound to PI or PC has given us an insight into how these proteins are able to facilitate the transfer of lipids. What needs to be deciphered is whether PITP proteins simply deliver PI (and PC) as transporters or function in some other mechanism. It is also important to find out how the PI and/or PC binding and transfer activity is connected to the variable biological functions that individual PITPs support. Many experiments (Cockcroft 2001; Cockcroft and Carvou 2007) using permeabilized cells firmly established the connection between PITPs and an increased production of phosphoinositides. The roles of phosphoinositides cannot be underestimated (Di Paolo and De Camilli 2006; Cockcroft and Carvou 2007;); these range from phospholipase C signaling, regulated exocytosis, vesicle formation to the regulation of the cell cycle. The hypothesis that PITPs function by providing PI for phosphorylation by various PI kinases in a spatially and time-regulated manner is the common mechanism behind the diverse functions of PITP proteins certainly requires attention. Unfortunately, monitoring of lipid transfer in intact cells is technically extremely challenging and has not yet been achieved for any lipid transporter to date.

Clearly, a great deal of progress has been made in understanding the role of LTPs in the physiology of eukaryotic cells. Still there is a wide gap between the biochemistry of 
these proteins and the detrimental phenotypes observed when these proteins are mutated or missing. Some of the remaining problems are: how are these proteins targeted to the proper subcellular membranes; how do they associate with membranes and how do they extract lipids from membranes; how do they distinguish between different lipids; what are the other components of the signaling pathways involving LTPs; do at least some of the LTPs serve as lipid sensors possibly regulating lipid metabolism; and to which extend binding of individual lipid molecules influences the function of PITPs.

\section{Conclusion}

Lipids, considered initially to be the building material of membranes and the way to store energy are now recognized as a source for the plethora of signaling molecules. The understanding of the role lipids play in the physiology of a eukaryotic cell may help us to understand the mechanisms behind lipid-related human diseases and devise strategies for better diagnosis and treatment. The molecular mechanism behind some of these diseases is known, whereas in some cases the link between the defect in lipid homeostasis and the disease pathology is less clear. We believe that to better understand the underlying role of lipids in pathological processes, in addition to studying these diseases directly, it is also of fundamental importance to study the basic and general mechanism of lipid homeostasis, lipid-protein interactions and biophysical properties of membrane lipids. Novel lipidomics technologies (Wolf and Quinn 2008) coupled with proteomics and genomics are now available to study lipids and their interactions with other cellular processes. Almost certainly, the combination of genetic, biochemical and structural analyses now available will lead to a better understanding of lipid metabolism and subsequently to better diagnosis and treatment of lipid-related diseases.

Acknowledgements. We wish to thank Roman Holič for critical reading of the manuscript and Mária Balážová and Peter Kohút for help with figures. Work in authors' laboratory related to cardiolipin metabolism and PITPs is supported by grants from the Slovak Research and Development Agency LPP-0291-09, APVV-0681-07, VVCE 0064-07, and by research grant VEGA 2/0077/10. K. P. and P. G. have no conflict of interest and no financial interest in the publication of this manuscript.

\section{References}

Acehan D., Khuchua Z., Houtkooper R. H., Malhotra A., Kaufman J., Vaz F. M., Ren M., Rockman H. A., Stokes D. L., Schlame M. (2009): Distinct effects of tafazzin deletion in differentiated and undifferentiated mitochondria. Mitochondrion 9, 86-95 doi:10.1016/j.mito.2008.12.001

Alb J. G., Jr., Phillips S. E., Rostand K., Cui X., Pinxteren J., Cotlin L., Manning T., Guo S., York J. D., Sontheimer H., Collawn J. F., Bankaitis V. A. (2002): Genetic ablation of phosphatidylinositol transfer protein function in murine embryonic stem cells. Mol. Biol. Cell. 13, 739-754

doi:10.1091/mbc.01-09-0457

Alb J. G., Jr., Cortese J. D., Phillips S. E., Albin R. L., Nagy T. R., Hamilton B. A., Bankaitis V. A. (2003): Mice lacking phosphatidylinositol transfer protein-alpha exhibit spinocerebellar degeneration, intestinal and hepatic steatosis, and hypoglycemia. J. Biol. Chem. 278, 33501-33518 doi:10.1074/jbc.M303591200

Bankaitis V. A., Malehorn D. E., Emr S. D., Greene R. (1989): The Saccharomyces cerevisiae SEC14 gene encodes a cytosolic factor that is required for transport of secretory proteins from the yeast Golgi complex. J. Cell. Biol. 108, 1271-1281 doi:10.1083/jcb.108.4.1271

Bankaitis V. A., Aitken J. R., Cleves A. E., Dowhan W. (1990): An essential role for a phospholipid transfer protein in yeast Golgi function. Nature 347, 561-562 doi:10.1038/347561a0

Bankaitis V. A., Mousley C. J., Schaaf G. (2010): The Sec14 superfamily and mechanisms for crosstalk between lipid metabolism and lipid signaling. Trends Biochem. Sci. 35, 150-160 doi:10.1016/j.tibs.2009.10.008

Barth P. G., Scholte H. R., Berden J. A., Van der Klei-Van Moorsel J. M., Luyt-Houwen I. E., Van ,t Veer-Korthof E. T., Van der Harten J. J., Sobotka-Plojhar M. A. (1983): An X-linked mitochondrial disease affecting cardiac muscle, skeletal muscle and neutrophil leucocytes. J. Neurol. Sci. 62, 327-355 doi:10.1016/0022-510X(83)90209-5

Barth P. G., Wanders R. J., Vreken P., Janssen E. A., Lam J., Baas F. (1999): X-linked cardioskeletal myopathy and neutropenia (Barth syndrome) (MIM 302060). J. Inherit. Metab. Dis. 22, 555-567 doi:10.1023/A:1005568609936

Barth P. G., Valianpour F., Bowen V. M., Lam J., Duran M., Vaz F. M., Wanders R. J. (2004): X-linked cardioskeletal myopathy and neutropenia (Barth syndrome): an update. Am. J. Med. Genet. A 126A, 349-354 doi:10.1002/ajmg.a.20660

Beranek A., Rechberger G., Knauer H., Wolinski H., Kohlwein S. D., Leber R. (2009): Identification of a cardiolipin-specific phospholipase encoded by the gene CLD1 (YGR110W) in yeast. J. Biol. Chem. 284, 11572-11578

doi:10.1074/jbc.M805511200

Bione S., D'Adamo P., Maestrini E., Gedeon A. K., Bolhuis P. A., Toniolo D. (1996): A novel X-linked gene, G4.5. is responsible for Barth syndrome. Nat. Genet. 12, 385-389 doi:10.1038/ng0496-385

Bomar J. M., Benke P. J., Slattery E. L., Puttagunta R., Taylor L. P., Seong E., Nystuen A., Chen W., Albin R. L., Patel P. D., Kittles R. A., Sheffield V. C., Burmeister M. (2003): Mutations in a novel gene encoding a CRAL-TRIO domain cause human Cayman ataxia and ataxia/dystonia in the jittery mouse. Nat. Genet. 35, 264-269 doi: $10.1038 /$ ng 1255 
Cantlay A. M., Shokrollahi K., Allen J. T., Lunt P. W., NewburyEcob R. A., Steward C. G. (1999): Genetic analysis of the G4.5 gene in families with suspected Barth syndrome. J. Pediatr. 135, 311-315 doi:10.1016/S0022-3476(99)70126-5

Chance B., Sies H., Boveris A. (1979): Hydroperoxide metabolism in mammalian organs. Physiol. Rev. 59, 527-605

Chen J., Delannoy M., Odwin S., He P., Trush M. A., Yager J. D. (2003): Enhanced mitochondrial gene transcript, ATP, bcl-2 protein levels, and altered glutathione distribution in ethinyl estradiol-treated cultured female rat hepatocytes. Toxicol. Sci. 75, 271-278 doi:10.1093/toxsci/kfg183

Chen S., He Q., Greenberg M. L. (2008): Loss of tafazzin in yeast leads to increased oxidative stress during respiratory growth. Mol. Microbiol. 68, 1061-1072 doi:10.1111/j.1365-2958.2008.06216.x

Chernomordik L. V., Kozlov M. M. (2005): Membrane hemifusion: crossing a chasm in two leaps. Cell 123, 375-382 doi:10.1016/j.cell.2005.10.015

Chernomordik L. V., Zimmerberg J., Kozlov M. M. (2006): Membranes of the world unite! J. Cell. Biol. 175, 201-207 doi:10.1083/jcb.200607083

Chicco A. J., Sparagna G. C. (2007): Role of cardiolipin alterations in mitochondrial dysfunction and disease. Am. J. Physiol. Cell Physiol. 292, C33-44 doi:10.1152/ajpcell.00243.2006

Christodoulou J., McInnes R. R., Jay V., Wilson G., Becker L. E., Lehotay D. C., Platt B. A., Bridge P. J., Robinson B. H., Clarke J. T. (1994): Barth syndrome: clinical observations and genetic linkage studies. Am. J. Med. Genet. 50, 255-264 doi:10.1002/ajmg.1320500309

Cichowski K., Jacks T. (2001): NF1 tumor suppressor gene function: narrowing the GAP. Cell 104, 593-604 doi:10.1016/S0092-8674(01)00245-8

Claypool S. M., Boontheung P., McCaffery J. M., Loo J. A., Koehler C. M. (2008): The cardiolipin transacylase, tafazzin, associates with two distinct respiratory components providing insight into Barth syndrome. Mol. Biol. Cell. 19, 5143-5155 doi:10.1091/mbc.E08-09-0896

Cleves A. E., Novick P. J., Bankaitis V. A. (1989): Mutations in the SAC1 gene suppress defects in yeast Golgi and yeast actin function. J. Cell. Biol. 109, 2939-2950 doi:10.1083/jcb.109.6.2939

Cleves A. E., McGee T. P., Whitters E. A., Champion K. M., Aitken J. R., Dowhan W., Goebl M., Bankaitis V. A. (1991): Mutations in the CDP-choline pathway for phospholipid biosynthesis bypass the requirement for an essential phospholipid transfer protein. Cell 64, 789-800 doi:10.1016/0092-8674(91)90508-V

Cockcroft S. (1998): Phosphatidylinositol transfer proteins: a requirement in signal transduction and vesicle traffic. Bioessays. 20, $423-432$

doi:10.1002/(SICI) 1521-1878(199805)20:5<423::AID-BIES9>3.0.CO;2-O

Cockcroft S. (2001): Phosphatidylinositol transfer proteins couple lipid transport to phosphoinositide synthesis. Semin. Cell Dev. Biol. 12, 183-191 doi: $10.1006 / \mathrm{scdb} .2000 .0235$

Cockcroft S., Carvou N. (2007): Biochemical and biological functions of class I phosphatidylinositol transfer proteins. Biochim. Biophys. Acta. 1771, 677-691

Costa V., Amorim M. A., Reis E., Quintanilha A., Moradas-Ferreira P. (1997): Mitochondrial superoxide dismutase is essential for ethanol tolerance of Saccharomyces cerevisiae in the post-diauxic phase. Microbiology 143 (Pt 5), 1649-1656 doi:10.1099/00221287-143-5-1649

Costa V., Moradas-Ferreira P. (2001): Oxidative stress and signal transduction in Saccharomyces cerevisiae: insights into ageing, apoptosis and diseases. Mol. Aspects Med. 22, 217-246 doi:10.1016/S0098-2997(01)00012-7

Cunningham E., Tan S. K., Swigart P., Hsuan J., Bankaitis V., Cockcroft S. (1996): The yeast and mammalian isoforms of phosphatidylinositol transfer protein can all restore phospholipase C-mediated inositol lipid signaling in cytosol-depleted RBL-2H3 and HL-60 cells. Proc. Natl. Acad. Sci. USA 93, 6589-6593 doi:10.1073/pnas.93.13.6589

D‘Angelo I., Welti S., Bonneau F., Scheffzek K. (2006): A novel bipartite phospholipid-binding module in the neurofibromatosis type 1 protein. EMBO Rep. 7, 174-179 doi:10.1038/sj.embor.7400602

De Vries K. J., Westerman J., Bastiaens P. I., Jovin T. M., Wirtz K. W., Snoek G. T. (1996): Fluorescently labeled phosphatidylinositol transfer protein isoforms (alpha and beta), microinjected into fetal bovine heart endothelial cells, are targeted to distinct intracellular sites. Exp. Cell Res. 227, 33-39 doi:10.1006/excr.1996.0246

Di Paolo G., De Camilli P. (2006): Phosphoinositides in cell regulation and membrane dynamics. Nature 443, 651-657 doi: $10.1038 /$ nature 05185

Du X., Takagi H. (2007): N-Acetyltransferase Mpr1 confers ethanol tolerance on Saccharomyces cerevisiae by reducing reactive oxygen species. Appl. Microbiol. Biotechnol. 75, $1343-1351$ doi:10.1007/s00253-007-0940-X

Fang M., Kearns B. G., Gedvilaite A., Kagiwada S., Kearns M., Fung M. K., Bankaitis V. A. (1996): Kes1p shares homology with human oxysterol binding protein and participates in a novel regulatory pathway for yeast Golgi-derived transport vesicle biogenesis. EMBO J. 15, 6447-6459

Griac P., Holic R., Tahotna D. (2006): Phosphatidylinositol-transfer protein and its homologues in yeast. Biochem. Soc. Trans. 34, 377-380 doi:10.1042/BST0340377

Gu Z., Valianpour F., Chen S., Vaz F. M., Hakkaart G. A., Wanders R. J., Greenberg M. L. (2004): Aberrant cardiolipin metabolism in the yeast taz1 mutant: a model for Barth syndrome. Mol. Microbiol. 51, 149-158 doi:10.1046/j.1365-2958.2003.03802.x

Hama H., Schnieders E. A., Thorner J., Takemoto J. Y., DeWald D. B. (1999): Direct involvement of phosphatidylinositol 4phosphate in secretion in the yeast Saccharomyces cerevisiae. J. Biol. Chem. 274, 34294-34300 doi:10.1074/jbc.274.48.34294 
Hamilton B. A., Smith D. J., Mueller K. L., Kerrebrock A. W., Bronson R. T., van Berkel V., Daly M. J., Kruglyak L., Reeve M. P., Nemhauser J. L., Hawkins T. L., Rubin E. M., Lander E. S. (1997): The vibrator mutation causes neurodegeneration via reduced expression of PITP alpha: positional complementation cloning and extragenic suppression. Neuron 18, 711-722 doi:10.1016/S0896-6273(00)80312-8

Hastings R., Steward C., Tsai-Goodman B., Newbury-Ecob R. (2009): Dysmorphology of Barth syndrome. Clin. Dysmorphol. 18, 185-187 doi:10.1097/MCD.0b013e32832a9e62

Hauff K. D., Hatch G. M. (2006): Cardiolipin metabolism and Barth Syndrome. Prog. Lipid Res. 45, 91-101 doi:10.1016/j.plipres.2005.12.001

Holthuis J. C., Levine T. P. (2005): Lipid traffic: floppy drives and a superhighway. Nat. Rev. Mol. Cell Biol. 6, 209-220 doi:10.1038/nrm1591

Imai H., Koumura T., Nakajima R., Nomura K., Nakagawa Y. (2003): Protection from inactivation of the adenine nucleotide translocator during hypoglycaemia-induced apoptosis by mitochondrial phospholipid hydroperoxide glutathione peroxidase. Biochem. J. 371, 799-809 doi:10.1042/BJ20021342

Jiang F., Ryan M. T., Schlame M., Zhao M., Gu Z., Klingenberg M., Pfanner N., Greenberg M. L. (2000): Absence of cardiolipin in the crd1 null mutant results in decreased mitochondrial membrane potential and reduced mitochondrial function. J. Biol. Chem. 275, 22387-22394 doi:10.1074/jbc.M909868199

Johnston J., Kelley R. I., Feigenbaum A., Cox G. F., Iyer G. S., Funanage V. L., Proujansky R. (1997): Mutation characterization and genotype-phenotype correlation in Barth syndrome. Am. J. Hum. Genet. 61, 1053-1058 doi: $10.1086 / 301604$

Kelley R. I., Cheatham J. P., Clark B. J., Nigro M. A., Powell B. R., Sherwood G. W., Sladky J. T., Swisher W. P. (1991): X-linked dilated cardiomyopathy with neutropenia, growth retardation, and 3-methylglutaconic aciduria. J. Pediatr. 119, 738-747 doi:10.1016/S0022-3476(05)80289-6

Koshkin V., Greenberg M. L. (2000): Oxidative phosphorylation in cardiolipin-lacking yeast mitochondria. Biochem. J. $347 \mathrm{Pt}$ 3, 687-691 doi:10.1042/0264-6021:3470687

LeBlanc M. A., McMaster C. R. (2010): Surprising roles for phospholipid binding proteins revealed by high throughput genetics. Biochem. Cell Biol. 88, 565-574

doi:10.1139/O09-171

Lev S. (2010) Non-vesicular lipid transport by lipid-transfer proteins and beyond. Nature Rev. Mol. Cell Biol. 11, 739-750 doi:10.1038/nrm2971

Levine T., Loewen C. (2006): Inter-organelle membrane contact sites: through a glass, darkly. Curr. Opin. Cell Biol. 18, 371-378 doi:10.1016/j.ceb.2006.06.011

Li G., Chen S., Thompson M. N., Greenberg M. L. (2007): New insights into the regulation of cardiolipin biosynthesis in yeast: implications for Barth syndrome. Biochim. Biophys. Acta. 1771, 432-441
Ma L., Vaz F. M., Gu Z., Wanders R. J., Greenberg M. L. (2004): The human TAZ gene complements mitochondrial dysfunction in the yeast taz1 delta mutant. Implications for Barth syndrome. J. Biol. Chem. 279, 44394-44399

doi:10.1074/jbc.M405479200

Macchioni L., Corazzi T., Davidescu M., Francescangeli E., Roberti R., Corazzi L. (2010): Cytochrome c redox state influences the binding and release of cytochrome $\mathrm{c}$ in model membranes and in brain mitochondria. Mol. Cell. Biochem. 341, 149-157 doi:10.1007/s11010-010-0446-1

Maw M. A., Kennedy B., Knight A., Bridges R., Roth K. E., Mani E. J., Mukkadan J. K., Nancarrow D., Crabb J. W., Denton M. J. (1997): Mutation of the gene encoding cellular retinaldehydebinding protein in autosomal recessive retinitis pigmentosa. Nat. Genet. 17, 198-200 doi:10.1038/ng1097-198

McLean L. R., Phillips M. C. (1984): Kinetics of phosphatidylcholine and lysophosphatidylchoine exchange between unilamellar vesicles. Biochemistry 23, 4624-4630 doi:10.1021/bi00315a017

Meier R., Tomizaki T., Schulze-Briese C., Baumann U., Stocker A. (2003): The molecular basis of vitamin E retention: structure of human alpha-tocopherol transfer protein. J. Mol. Biol. 331, 725-734 doi:10.1016/S0022-2836(03)00724-1

Milligan S. C., Alb J. G., Jr., Elagina R. B., Bankaitis V. A., Hyde D. R. (1997): The phosphatidylinositol transfer protein domain of Drosophila retinal degeneration B protein is essential for photoreceptor cell survival and recovery from light stimulation. J. Cell. Biol. 139, 351-363 doi:10.1083/jcb.139.2.351

Min K. C., Kovall R. A., Hendrickson W. A. (2003): Crystal structure of human alpha-tocopherol transfer protein bound to its ligand: implications for ataxia with vitamin E deficiency. Proc. Natl. Acad. Sci. U.S.A. 100, 14713-14718 doi:10.1073/pnas.2136684100

Murray J. P., McMaster C. R. (2005): Nte1p-mediated deacylation of phosphatidylcholine functionally interacts with Sec14p. J. Biol. Chem. 280, 8544-8552 doi:10.1074/jbc.M413999200

Ouahchi K., Arita M., Kayden H., Hentati F., Ben Hamida M., Sokol R., Arai H., Inoue K., Mandel J. L., Koenig M. (1995): Ataxia with isolated vitamin E deficiency is caused by mutations in the alpha-tocopherol transfer protein. Nat. Genet. 9, 141-145 doi:10.1038/ng0295-141

Pfeiffer K., Gohil V., Stuart R. A., Hunte C., Brandt U., Greenberg M. L., Schagger H. (2003): Cardiolipin stabilizes respiratory chain supercomplexes. J. Biol. Chem. 278, 52873-52880 doi:10.1074/jbc.M308366200

Sha B., Phillips S. E., Bankaitis V. A., Luo M. (1998): Crystal structure of the Saccharomyces cerevisiae phosphatidylinositoltransfer protein. Nature 391, 506-510 doi: $10.1038 / 35179$

Schaaf G., Ortlund E. A., Tyeryar K. R., Mousley C. J., Ile K. E., Garrett T. A., Ren J., Woolls M. J., Raetz C. R., Redinbo M. R., Bankaitis V. A. (2008): Functional anatomy of phospholipid 
binding and regulation of phosphoinositide homeostasis by proteins of the sec14 superfamily. Mol. Cell 29, 191-206 doi:10.1016/j.molcel.2007.11.026

Schlame M., Rua D., Greenberg M. L. (2000): The biosynthesis and functional role of cardiolipin. Prog. Lipid Res. 39, 257-288 doi:10.1016/S0163-7827(00)00005-9

Schlame M., Towbin J. A., Heerdt P. M., Jehle R., DiMauro S., Blanck T. J. (2002): Deficiency of tetralinoleoyl-cardiolipin in Barth syndrome. Ann. Neurol. 51, 634-637 doi:10.1002/ana.10176

Schmidt M. R., Birkebaek N., Gonzalez I., Sunde L. (2004): Barth syndrome without 3-methylglutaconic aciduria. Acta Paediatr. 93, 419-421 doi:10.1111/j.1651-2227.2004.tb02974.x

Spencer C. T., Byrne B. J., Gewitz M. H., Wechsler S. B., Kao A. C., Gerstenfeld E. P., Merliss A. D., Carboni M. P., Bryant R. M. (2005): Ventricular arrhythmia in the X-linked cardiomyopathy Barth syndrome. Pediatr. Cardiol. 26, 632-637 doi:10.1007/s00246-005-0873-z

Sreenivas A., Patton-Vogt J. L., Bruno V., Griac P., Henry S. A. (1998): A role for phospholipase D (Pld1p) in growth, secretion, and regulation of membrane lipid synthesis in yeast. J. Biol. Chem. 273, 16635-16638 doi:10.1074/jbc.273.27.16635

Steward C. G., Newbury-Ecob R. A., Hastings R., Smithson S. F., Tsai-Goodman B., Quarrell O. W., Kulik W., Wanders R., Pennock M., Williams M., Cresswell J. L., Gonzalez I. L., Brennan P. (2010): Barth syndrome: an X-linked cause of fetal cardiomyopathy and stillbirth. Prenat. Diagn. 30, 970-976 doi: $10.1002 / p d .2599$

Tanaka S., Hosaka K. (1994): Cloning of a cDNA encoding a second phosphatidylinositol transfer protein of rat brain by complementation of the yeast sec 14 mutation. J. Biochem. 115, 981-984

Turrens J. F. (2003): Mitochondrial formation of reactive oxygen species. J. Physiol. 552, 335-344 doi:10.1113/jphysiol.2003.049478

Vaz F. M., Houtkooper R. H., Valianpour F., Barth P. G., Wanders R. J. (2003): Only one splice variant of the human TAZ gene encodes a functional protein with a role in cardiolipin metabolism. J. Biol. Chem. 278, 43089-43094 doi:10.1074/jbc.M305956200

Vreken P., Valianpour F., Nijtmans L. G., Grivell L. A., Plecko B., Wanders R. J., Barth P. G. (2000): Defective remodeling of cardiolipin and phosphatidylglycerol in Barth syndrome. Biochem. Biophys. Res. Commun. 279, 378-382 doi:10.1006/bbrc.2000.3952
Wang X. (2001): The expanding role of mitochondria in apoptosis. Genes Dev. 15, 2922-2933

Welti S., Fraterman S., D'Angelo I., Wilm M., Scheffzek K. (2007): The sec14 homology module of neurofibromin binds cellular glycerophospholipids: mass spectrometry and structure of a lipid complex. J. Mol. Biol. 366, 551-562 doi:10.1016/j.jmb.2006.11.055

Wirtz K. W. (1991): Phospholipid transfer proteins. Annu. Rev. Biochem. 60, 73-99 doi:10.1146/annurev.bi.60.070191.000445

Wolf C., Quinn P. J. (2008): Lipidomics: practical aspects and applications. Prog. Lipid Res. 47, 15-36

Wu W. I., Routt S., Bankaitis V. A., Voelker D. R. (2000): A new gene involved in the transport-dependent metabolism of phosphatidylserine, PSTB2/PDR17, shares sequence similarity with the gene encoding the phosphatidylinositol/phosphatidylcholine transfer protein, SEC14. J. Biol. Chem. 275, 14446-14456 doi:10.1074/jbc.275.19.14446

Wu W. I., Voelker D. R. (2001): Characterization of phosphatidylserine transport to the locus of phosphatidylserine decarboxylase 2 in permeabilized yeast. J. Biol. Chem. 276, 7114-7121 doi:10.1074/jbc.M010278200

Xie Z., Fang M., Rivas M. P., Faulkner A. J., Sternweis P. C., Engebrecht J. A., Bankaitis V. A. (1998): Phospholipase D activity is required for suppression of yeast phosphatidylinositol transfer protein defects. Proc. Natl. Acad. Sci. U.S.A. 95, 12346-12351 doi:10.1073/pnas.95.21.12346

Xie Z., Fang M., Bankaitis V. A. (2001): Evidence for an intrinsic toxicity of phosphatidylcholine to Sec14p-dependent protein transport from the yeast Golgi complex. Mol. Biol. Cell 12, $1117-1129$

Xu Y., Condell M., Plesken H., Edelman-Novemsky I., Ma J., Ren M., Schlame M. (2006a): A Drosophila model of Barth syndrome. Proc. Natl. Acad. Sci. U.S.A. 103, 11584-11588 doi:10.1073/pnas.0603242103

Xu Y., Malhotra A., Ren M., Schlame M. (2006b): The enzymatic function of tafazzin. J. Biol. Chem. 281, 39217-39224 doi:10.1074/jbc.M606100200

Zhang M., Mileykovskaya E., Dowhan W. (2002): Gluing the respiratory chain together. Cardiolipin is required for supercomplex formation in the inner mitochondrial membrane. J. Biol. Chem. 277, 43553-43556 doi:10.1074/jbc.C200551200

Received: November 11, 2010

Final version accepted: January 13, 2011 